\title{
Optimal Economic Stabilization Policy: An Extended Framework
}

\section{Citation}

Friedman, Benjamin Morton. 1972. Optimal economic stabilization policy: An extended framework. Journal of Political Economy 80(5): 1002-1022.

\section{Published Version}

doi:10.1086/259947

\section{Permanent link}

http://nrs.harvard.edu/urn-3:HUL.InstRepos:4554308

\section{Terms of Use}

This article was downloaded from Harvard University's DASH repository, and is made available under the terms and conditions applicable to Other Posted Material, as set forth at http:// nrs.harvard.edu/urn-3:HUL.InstRepos:dash.current.terms-of-use\#LAA

\section{Share Your Story}

The Harvard community has made this article openly available.

Please share how this access benefits you. Submit a story.

Accessibility 


\title{
Optimal Economic Stabilization Policy: An Extended Framework
}

\section{Benjamin M. Friedman}

Morgan Stanley \& Co.

\begin{abstract}
This paper outlines an optimization framework which extends the familiar Tinbergen-Theil model in two ways. First, a "piecewise quadratic" replaces the standard quadratic objective function. Second, the time horizon of the optimization becomes, within the context of economic stabilization problems, endogenous to the optimization process itself. The purpose of both extensions is to escape the conceptual restrictiveness of the Tinbergen-Theil structure while preserving the practical convenience of that model for applied policy work. The paper also describes a solution algorithm incorporating these two extensions, and it presents the results of a sample computational application based on the $1957-58$ recession.
\end{abstract}

The goal of mitigating economic fluctuations and their social effects has long attracted economists' interest, and it continues to do so. After nearly a decade of seemingly perpetual expansion in the United States economy, the 1970 recession has once more focused attention on stabilization policy. How can policy cope with the apparently conflicting goals of highemployment prosperity and price stability? How rapidly should policy seek to return the economy to a full-employment situation? What timing patterns should the fiscal and monetary policy authorities adopt for their actions? These and similar questions form the basis of discussions on the academic, political, and popular levels.

The post-World War II economics literature has developed-in the work of Theil (1964), Tinbergen (1966), and others ${ }^{1}$ - at least one practi-

An earlier version of this paper was presented at the Second World Congress of the Econometric Society, Cambridge, England, September 1970. I am grateful for support from the Harvard University Society of Fellows and for helpful comments from Martin S. Feldstein and Dale W. Jorgenson.

${ }^{1}$ For additional contributions, see Hickman (1965) and Fox, Sengupta, and Thorbecke (1966). 
cal, convenient framework for planning quantitative aggregate policy to cope with such fluctuations; this is the familiar dynamic optimization of a quadratic criterion function subject to linear equality constraints. Nevertheless, the conceptual restrictiveness of this framework prevents it from being widely useful in actual applications to policy formulation. The quadratic criterion function, for example, does not seem to offer a very good representation of policy makers' preferences; and the necessity of an arbitrary choice of time horizon prevents using the framework to answer several significant policy questions.

At the same time, the more generalized mathematical programming ${ }^{2}$ and systems-control ${ }^{3}$ literatures have, on the whole, had too general a presentation to be of great use ir economic policy applications. The principles enunciated within the various divisions of optimization research are of great relevance and importance in such problems, but the difficulty of adapting these principles remains a significant obstacle to their application to economic policy problems and in particular to economic stabilization. Much "bridge" work remains to be done to select that part of the generality which is of sufficient potential value to keep, as well as to render it readily usable in an operational and computational sense. This paper is, at least in part, an attempt along such lines.

This paper outlines an optimization framework which extends the Tinbergen-Theil model in two ways. First, a "piecewise quadratic" replaces the standard quadratic criterion function. Second, the time horizon of the optimization becomes, within the context of economic stabilization problems, endogenous to the optimization process itself. The object of both extensions is to escape the conceptual restrictiveness of the Tinbergen-Theil structure while preserving its practical convenience for applied policy work. To focus clearly on these two extensions, this paper deals with a deterministic system in which the potential impact of policy actions on the economy is known with certainty. ${ }^{4}$

Section I discusses the piecewise quadratic criterion function. Section II discusses the endogenous time horizon and the associated concept of the "policy interval." Section III outlines the solution algorithm for the optimization and analyzes the interaction of these two extensions. Section

2 See, for example, Pontryagin et al. (1962), Fan and Wang (1964), and Mangasarian (1969).

${ }^{3}$ See, for example, Sage (1968) and Bryson and Ho (1969).

4 Although this paper does not discuss the implications of uncertainty, it is best to state at the outset that the piecewise quadratic criterion function cannot yield the first-period certainty equivalence result which Simon (1956) and Theil (1957) have derived for quadratic criteria. Given the current state of economic knowledge, however, the sacrifice of this property is not a major consideration for applied policy work, since the Simon-Theil result requires perfect knowledge of the parameters which reflect the impact of policy instruments on policy targets (that is, elements of matrix $\boldsymbol{R}$ in eq. [2] below) and permits uncertainty to enter the problem in a linear fashion only. 
IV presents the results of a sample computational application of the algorithm. Section V briefly summarizes the optimization methods offered.

\section{The Piecewise Quadratic Criterion Function}

A standard form of criterion function for optimization problems is the quadratic

$$
W(x, y)=a^{\prime} x+b^{\prime} y+\frac{1}{2} \cdot\left(x^{\prime} A x+y^{\prime} B y+x^{\prime} C y+y^{\prime} C^{\prime} x\right)
$$

in which vector $x$ represents values of instrument variables, subject to control, vector $y$ represents values of target variables, related to the instruments by a set of linear constraints

$$
\boldsymbol{Y}=\boldsymbol{R} \boldsymbol{x}+\boldsymbol{s}
$$

and $a, b, A, B$, and $C$ are conformable vectors and matrices of previously established coefficients. ${ }^{5}$

For expositional simplicity (involving no loss of generality), treat $a$ and $\boldsymbol{b}$ as null vectors, $\boldsymbol{C}$ as a null matrix, and $\boldsymbol{A}$ and $\boldsymbol{B}$ as nonnegative diagonal matrices. Then for each variable $x_{i}$ or $y_{i}$, there is some desired value or zero-penalty point $x_{i}{ }^{*}$ or $y_{i}{ }^{*}$, and from the matrices $\boldsymbol{A}$ and $\boldsymbol{B}$ some penalty rate $a_{i i}$ or $b_{i i}$. The penalty attached to a given realization for any variable is then $\frac{1}{2} a_{i} \tilde{x}_{i}{ }^{2}$, where $\widetilde{x}_{i}=x_{i}-x_{i}{ }^{*}$, or $\frac{1}{2} b_{i i} \tilde{y}_{i}{ }^{2}$, where $\tilde{y}_{i}=y_{i}-y_{i}{ }^{*}$.

In applications to economic policy, however, the quadratic function is not a very satisfactory representation of preferences likely to be pursued by policy makers. Exact desired values $y_{i}{ }^{*}$ and $x_{i}{ }^{*}$ for given policy targets or instruments in any period may not exist in these real preferences; often, policy makers see certain variables more as constraints, in the sense of bearing an implicit loss only for values outside some range. An even more unrealistic aspect of the quadratic function is the requirement that deviations of a target or instrument variable from its desired value bear the same loss regardless of the direction of the deviation.

The piecewise quadratic function offers a more general framework for policy optimization. Specifically, it is in general asymmetrical and only convex (as opposed to strictly convex). In reality, it is three distinct functions, welded in such a way as to preserve those properties of the more restrictive quadratic form which are essential to the optimization process. The generalization achieved by this function relaxes the principal unattractive requirements associated with the quadratic form, and its inclusion within the framework of the Tinbergen-Theil approach therefore renders that approach more realistic and useful for examining economic policy problems.

\footnotetext{
5 Equations (1) and (2) represent a dynamic system in that $\boldsymbol{x}$ and $\boldsymbol{y}$ are stacked vectors, as Section III below emphasizes. Since this paper deals entirely with a deterministic system, matrix $\boldsymbol{R}$ and vector $\boldsymbol{s}$ are known with certainty in (2).
} 
For each target variable $y_{i}$ and instrument variable $x_{i}$ bearing piecewise quadratic treatment, two fixed values divide the entire range of possible values of the variable into three distinct convex sets. The middle range is a closed, bounded set of values which uniformly bear a zero loss. The two extreme ranges, sets bounded on one side only and not closed on either side, contain values which bear nonzero losses. Two quadratic functions, one defined for either extreme range, determine these losses according to independent patterns, constrained so that the functions approach limiting values of zero as the variables approach the boundaries of the middle range; these two functions are not defined for the boundary points.

Applied, for example, to the $\frac{1}{2} a_{i i} \tilde{x}_{i}^{2}$ terms in the sum $\frac{1}{2} \tilde{x}^{\prime} A \tilde{x}$ in criterion function (1), the piecewise quadratic generalization has two effects.

First, for each instrument variable $x_{i}$ bearing piecewise quadratic treatment, it replaces the constant $a_{i i}$ by the decision rule

where

$$
a_{i i}=\left[\begin{array}{lll}
a_{i i}{ }^{u} & \text { if } & x_{i} \in U\left(x_{i}\right)=\left\{x_{i} \mid x_{i}>x_{i}{ }^{u}\right\} \\
0 & \text { if } & x_{i} \in M\left(x_{i}\right)=\left\{x_{i} \mid x_{i}{ }^{l} \leqslant x_{i} \leqslant x_{i}{ }^{u}\right\} \\
a_{i i}{ }^{l} & \text { if } & x_{i} \in L\left(x_{i}\right)=\left\{x_{i} \mid x_{i}<x_{i}{ }^{l}\right\}
\end{array}\right.
$$

$a_{i i}{ }^{u}=$ criterion coefficient applying to values of $x_{i}$ in $U\left(x_{i}\right)$, the upper extreme set for $x_{i}$;

$a_{i i}{ }^{l}=$ criterion coefficient applying to values of $x_{i}$ in $L\left(x_{i}\right)$, the lower extreme set for $x_{i}$;

$x_{i}{ }^{u}=$ upper boundary point of $M\left(x_{i}\right)$, the middle set for $x_{i}$;

$x_{i}{ }^{l}=$ lower boundary point of $M\left(x_{i}\right)$.

Second, for each such $x_{i}$ the piecewise quadratic generalization replaces the $\tilde{x}_{i}=x_{i}-x_{i} *$ relation by the decision rule

$$
\tilde{x}_{i}=\left[\begin{array}{ccccc}
\left(x_{i}-x_{i}{ }^{u}\right) & & & \text { if } & x_{i} \epsilon U\left(x_{i}\right) \\
\left(x_{i}-x_{i}{ }^{u}\right) & \text { or } & \left(x_{i}{ }^{l}-x_{i}\right) & \text { if } & x_{i} \in M\left(x_{i}\right) \\
\left(x_{i}{ }^{l}-x_{i}\right) & & & \text { if } & x_{i} \in L\left(x_{i}\right),
\end{array}\right.
$$

where $x_{i}{ }^{u}, x_{i}{ }^{l}, U\left(x_{i}\right), M\left(x_{i}\right)$, and $L\left(x_{i}\right)$ are defined as for decision rule (3). The option in (4) for $x_{i}$ in the middle set is arbitrary, since $a_{i i}=0$ for these values.

Decision rules (3) and (4), taken together, yield a piecewise quadratic loss function element $\frac{1}{2} a_{i i} \widetilde{x}_{i}{ }^{2}$. Rules specifying values for $y_{i}{ }^{u}, y_{i}{ }^{l}, b_{i i}{ }^{u}$, and $b_{i i}{ }^{l}$ yield analogous functions $\frac{1}{2} b_{i i} \tilde{y}_{i}{ }^{2}$. Figure 1 , which plots a sample $\frac{1}{2} a_{i i} \widetilde{x}_{i}^{2}$ term on the vertical axis against $x_{i}$ on the horizontal axis, illustrates the asymmetrical (nonstrictly) convex properties of the basic functional form. ${ }^{6}$

${ }^{6}$ Figure 1 shows a function $\frac{1}{2} a_{i} \tilde{x}_{i}^{2}$ which may be "typical" in some sense but 


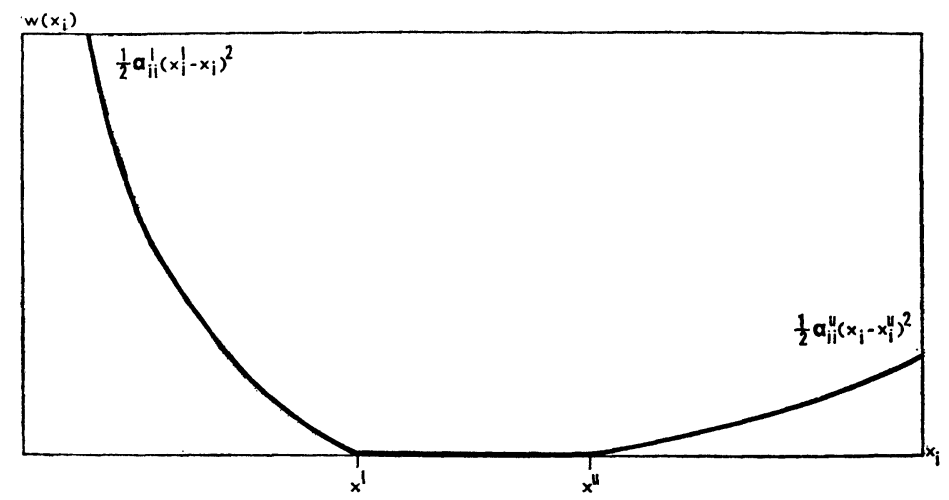

FIG. 1.-Prototypical piecewise quadratic function

The quadratic function is the special case of the piecewise quadratic in which both symmetry and strict convexity maintain. Symmetry requires $a_{i i}{ }^{u}=a_{i i}{ }^{l}$. Strict convexity requires $x_{i}{ }^{u}=x_{i}{ }^{l}$, that is, the collapse of the zero loss set $M\left(x_{i}\right)$ to a single point. Hence, for the quadratic function, constant $a_{i i}=a_{i i}{ }^{u}=a_{i i}{ }^{l}$ replaces decision rule (3) and constant $x_{i}{ }^{*}=$ $x_{i}{ }^{u}=x_{i}^{l}$ replaces decision rule (4).

In addition to its generality and flexibility, which permit a more realistic representation of economic policy preferences, the piecewise quadratic criterion function has at least two computational advantages. One concerns its interaction with the extension to the Tinbergen-Theil approach developed in Section II below; Section III discusses this interaction explicitly.

The second computational advantage arises in the context of the distinction between equality and inequality constraints. The Theil model optimizes the function (1) subject to a set of linear equality constraints (2) ; this model, unlike linear programming or general mathematical programming techniques, does not admit inequality constraints. The piecewise quadratic function facilitates incorporating inequality constraints while at the same time staying within the operationally convenient Theil framework. This inequality constraint capability is a further aspect of the piecewise quadratic function's more realistic representation of policy preferences. $^{7}$

which does not utilize the full flexibility of the piecewise quadratic form. Defining $x_{i}{ }^{u}=\infty$ or $x_{i}{ }^{l}=-\infty$, for example, specifies a function which assigns a loss to deviations of $x_{i}$ on one side only of a given value. The discussion below treats the analogous case for $a_{i i}{ }^{u}=\infty$ or $a_{i i}{ }^{l}=\infty$ or both.

${ }^{7} \mathrm{It}$ is possible to argue that, since piecewise quadratic criteria involve more individual parameters than do standard quadratics, the information required would be more difficult to extract from policy makers. Such an argument seems not to be the case. Precisely because the piecewise quadratic function is capable of representing policy preferences more realistically, the required information should be easier to ex- 

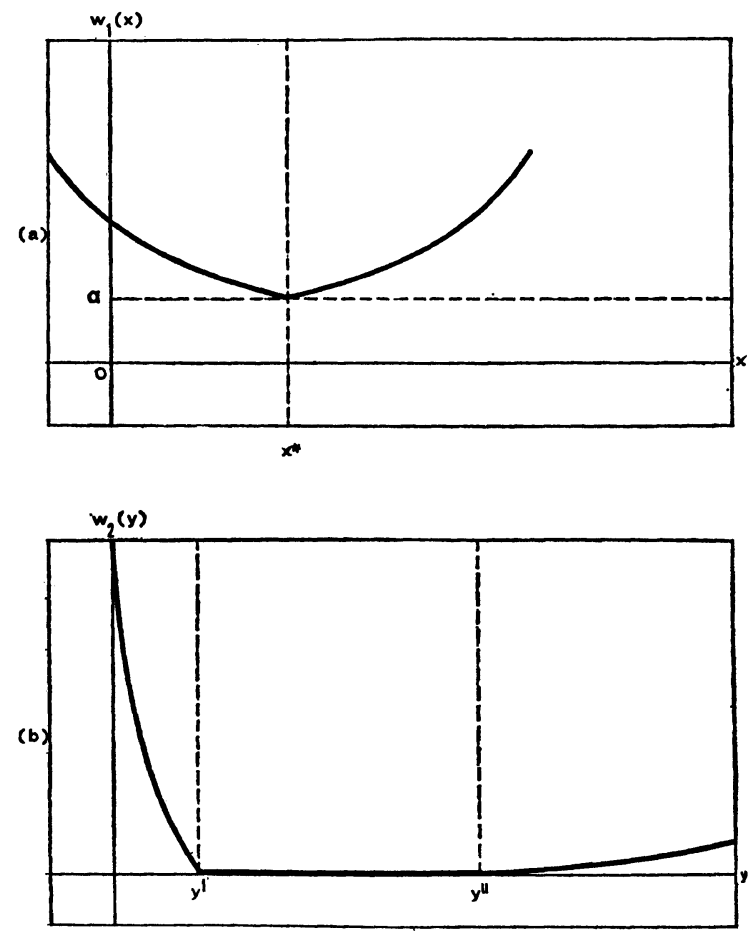

Fig. 2.-Functions $6(a)$ and $7(b)$

Setting $a_{i i}{ }^{u}=\infty$ imposes upon the optimization the constraint $x_{i} \leqslant$ $x_{i}{ }^{u}$; setting $a_{i i}{ }^{l}=\infty$ imposes the corresponding constraint $x_{i} \geqslant x_{i}{ }^{l}$. In computational practice, infinite values are not manageable for $a_{i i}{ }^{u}$ and $a_{i i}{ }^{l}$. Nevertheless, approximating infinity by values of $a_{i i}{ }^{u}$ or $a_{i i}{ }^{l}$ larger than the other elements $a_{i j}, b_{i j}$, and $c_{i j}$ by several orders of magnitude is an effective way to approximate the relevant inequality constraint in the solution to the piecewise quadratic optimization. ${ }^{8}$

Figures 2 and 3 show how individual piecewise quadratic terms combine to form the piecewise quadratic function for a given problem. For purposes of illustration, they treat a problem in which $x$ and $y$ are scalars. The preference function contains no term in the cross-product of $x$ and $y$ and so is separable:

$$
w(x, y)=w_{1}(x)+w_{2}(y),
$$

where $w_{1}(x)$ is a straightforward quadratic, while $w_{2}(y)$ is a piecewise quadratic function:

tract; it is no longer necessary to force preferences to fit into such an artificially limiting framework as the standard quadratic function.

8 This technique is the "penalty method" of nonlinear programming with constraints in Zangwill (1969), chap. 12. The basic idea is to approximate the feasible region from the outside. 


$$
w_{2}(y)=\left[\begin{array}{llc}
b_{1}(x)=a_{1} x^{2}+a_{2} x+a_{3}, \\
0 & \text { if } & y \in U(y)=\left\{y \mid y>y^{u}\right\} \\
b^{l}\left(y^{l}-y\right)^{2} & \text { if } & y \in M(y)=\left\{y \mid y^{l} \leqslant y \leqslant y^{u}\right\}
\end{array}\right.
$$

Figure 2 plots functions (6) and (7). The former is strictly convex for $a_{1}>0$ and has a minimum at $x^{*}=-a_{2} / 2 a_{1}$, where $w_{1}\left(x^{*}\right)=a=a_{3}-$ $\left(a_{2}\right)^{2} / 4 a_{1}$. The latter is convex for $b^{u}, b^{l}>0$, having a flat zero range for $y \in M(y)$. The curves as drawn imply $b^{l}>a_{1}>b^{u}$.

Since convexity (even when not strict) is preserved in the addition operation (Managasarian 1969, chap. 9), $w(x, y)$ in equation (5) is itself convex. Using a linear relation $y=R x+s$, for scalar $R$ and $s$, permits plotting equation (5) for values of $x$. Three cases emerge, depending upon which of the three sets of values of $y$ contains $\left(R x^{*}+s\right)$. Figure 3 plots $w(x, y)$ against $x$ for the three possible cases for $R>0$. In panel $(a), x_{1}<x^{*}$ $<x_{2}$, where $x_{1}=\left(y^{l}-s\right) / R$ and $x_{2}=\left(y^{u}-s\right) / R$; hence, the minimand is $\hat{x}=x^{*}$. In panel $(b), x^{*}<x_{1}$, and the minimand $\hat{x}$ satisfies $x^{*}<\hat{x}<$ $x_{1}$. The opposite case is that of panel $(c)$, in which $x^{*}>x_{2}$; here the minimand $\hat{x}$ satisfies $x_{2}<\hat{x}<x^{*}$.
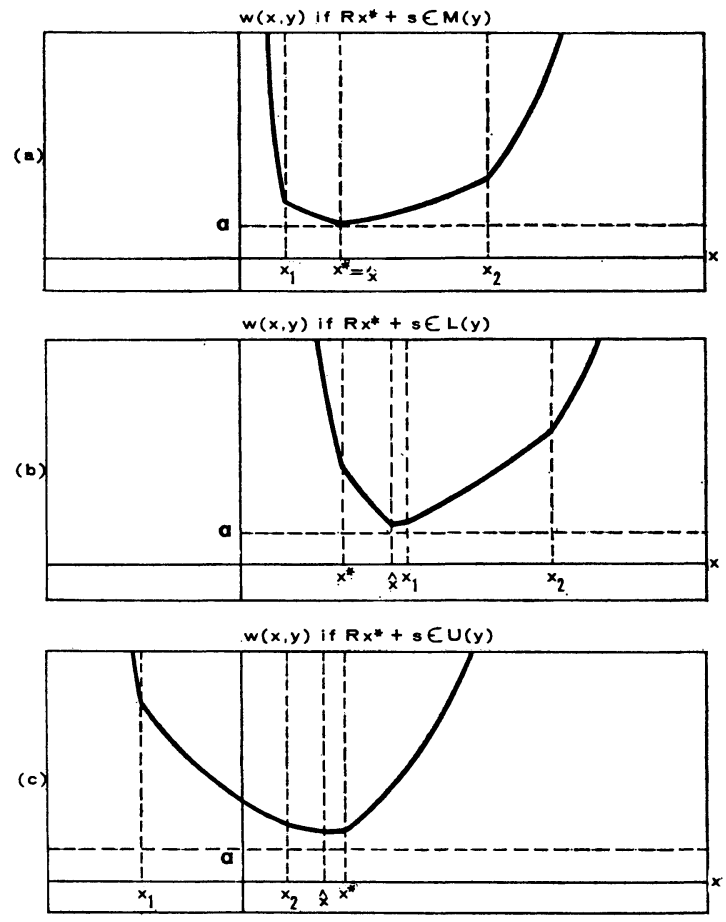

Fig. 3.-Three cases for function 5 


\section{The Endogenous Time Horizon}

A major shortcoming of the Tinbergen-Theil approach to optimization is that "the choice of $T$, the length of the horizon, is in many cases somewhat arbitrary." 9 Applications to economic stabilization policy are examples of such cases. A related gap in the Tinbergen-Theil approach is the omission of terminal conditions to apply at the close of the optimization time span in period $t=T$. Terminal conditions are more familiar in the control literature and that of dynamic programming; ${ }^{10}$ they are difficult to devise and to apply in a Theil solution. A single extension of the Tinbergen-Theil framework, based on a particular conception of the optimization problem in a stabilization context, facilitates avoiding both of these difficulties by a method which determines the appropriate time horizon endogenously within the optimization procedure.

The fundamental dynamic concept of the endogenous time horizon approach to optimization is the "policy interval," defined as that period of time during which specific stabilization policy is in effect.

This concept rests upon the notion of a stable economy, which in the short run strays from its "normal" path. Given such a deviation, the goal of the policy authorities is to return the economy to this path while minimizing specified costs associated with being away from the path. The discussion of zero loss ranges in Section I leads easily to the idea of identifying this long-run path as a set of ranges of acceptable values of key variables, rather than as a single set of required point values for each period in time.

The policy-interval concept also assumes the existence of some "normal" economic policy which pertains as long as the economy stays within the acceptable bounds of the long-run path. In cases of deviation, the policy authorities pursue objectives associated with returning to this path by implementing specific stabilization policy actions-hence the name "policy interval." When the economy has in fact returned to the long-run path, the stabilization element of economic policy terminates and the policy reverts to the appropriate long-run norm.

This intuitive and descriptive definition of the policy interval raises at least three questions. What determines whether the economy has deviated sufficiently from the long-run norm to identify the deviation as a policy interval? Once a policy interval has begun, what determines whether the economy has returned sufficiently to the long-run norm to end the policy

9 Theil (1964), p. 154. Theil offers the alternative approach of an infinite horizon, solved using infinite band matrices (chap. 5), as well as a moving horizon, which is the truncated case of an infinite horizon (pp. 154-61). The concept of the endogenous time horizon developed here is more closely related to Theil's finite horizon base, but, as the discussion indicates, it has more flexibility.

10 See, for example, the discussion of two-point boundary value problems in Bellman and Dreyfus (1962). 
interval? What goals do the particular stabilization policy actions within the policy interval pursue?

In response to these questions, these three properties more precisely identify a given policy interval: initial conditions, terminal conditions, and criterion function. These three elements of the optimization enter the analysis in the following manner.

The initial conditions specify the conditions which the economy must satisfy for a policy interval to begin. According to these initial conditions, the policy interval itself begins at that point in time when a particular economic variable (or set of variables) strays outside the given limits of the long-run acceptable range. Examples may be the unemployment rate or the rate of price increase rising too high, or the growth rate of real output falling too low. Beginning in the period which first satisfies the initial conditions for a policy interval, the authorities undertake specific stabilization policy actions.

If the time period used in the analysis is short, or if the relevant datareporting machinery entails long delays, problems may arise with the role of the initial conditions as specified above. Specifically, although the value of a variable in a given period may satisfy the appropriate initial conditions for a policy interval, this fact may not become apparent until some time later. Such cases require a reformulation of the role of the initial conditions to incorporate either a forecasting procedure or a lagged policy response.

The terminal conditions specify the conditions which the economy must satisfy for a policy interval in progress to come to an end, that is, for the authorities to revert to the long-run policy actions which pertain in the absence of specific stabilization efforts. The policy interval itself ends, that is, specific stabilization policy terminates, at that point in time when all relevant economic variables first satisfy the applicable terminal conditions. That particular period marks the time horizon of the optimization procedure which determines the optimal stabilization policy during the policy interval. ${ }^{11}$

A necessary part of the terminal conditions is the return to acceptability of the particular variable or variables which initiated the policy interval via the initial conditions, but the terminal conditions may involve other variables as well. In a policy interval initiated by an excessive unemployment rate, for example, typical terminal conditions may permit the policy interval to end only when both the unemployment rate and the rate of price inflation are within acceptable bounds; hence, a policy which returns

11 In the cases noted above, for which delays in data observation raise problems for the interpretation of the initial conditions, it is also necessary to reformulate the role of the terminal conditions to incorporate either a forecasting procedure or a delayed end of the policy interval. 
unemployment to its normal level, but only at the expense of inducing a price inflation, is not sufficient.

More generally formulated terminal conditions are also possible. In the example above of terminal conditions based on values of the unemployment rate and the rate of price inflation, satisfaction of specified conditions in one period only may for some applications be insufficient. In such cases, the terminal conditions may require values of the former variable in a given range for $K$ consecutive periods and values of the latter in a given range for $L$ consecutive periods, where in general $K \neq L$. An even more general extension of the terminal conditions is the requirement that the specific stabilization policies undertaken during the policy interval not induce particular undesirable effects after the policy interval's conclusion. ${ }^{12}$

The criterion function sets forth in a mathematical framework those economic goals which the authorities pursue during the policy interval. These goals may be, but are not necessarily, related to long-run goals associated with the long-run normal path of the economy. Just as it is necessary for the specific variable or variables in the initial conditions to appear in the terminal conditions, all variables in the terminal conditions must necessarily appear in the criterion function. The criterion function may, analogously, have additional arguments. It may be desirable, for example, to optimize some function of the balance-of-payments surplus during the policy interval, without necessarily making such a surplus a specific constraint by including it in the terminal conditions.

As one simple illustration of the mechanics of the policy-interval concept and the associated endogenous time horizon optimization, assume that the normal long-run growth rate of real output, $d X / d t$, is sufficiently in excess of $r$ to warrant identifying $r$ as the "minimum acceptable rate" of growth. The initial condition for a "recessionary" policy interval is then that $d X / d t$ falls below $r$. This initial condition means that an observed $d X / d t<r$ calls for stabilization policy actions; for $d X / d t \geqslant r$, policy maintains its long-run course which is independent of any immediate stabilization needs.

Once a policy interval has begun, it is possible to define a "minimum acceptable level" of real output by projecting forward, at the "minimum acceptable rate" $r$, the last observation of real output before the beginning of the policy interval. One possible terminal condition for this policy interval may then be that real output attains or exceeds this "minimum desired level." 13

12 Since all such effects of these policy actions are easily computable within the optimization procedure by using the $\boldsymbol{R}$ matrix, this extended form of terminal condition is in practice not difficult to apply.

13 The exercise in Section IV below uses a terminal condition constructed in this 
Figure 4 shows paths for actual and minimum desired real output as functions of time. Through period $t=0$, real output grows at $d X / d t \geqslant r$, and so the minimum desired path is unnecessary. In period $t=1$, however, $d X / d t<r$, which satisfies the initial condition. The minimum desired path is then an extrapolation at growth rate $r$ of the value of real output in period $t=0$. The effect of the specific stabilization policy actions, together with the inherent long-run stability of the economy, returns actual output in figure 4 to a value above the minimum desired level in period $t=\hat{T}$, thereby satisfying the terminal condition. $\hat{T}$ is then the time horizon of the policy-interval optimization problem.

Using the policy-interval concept in this way makes the time horizon an endogenous, simultaneously determined element of the optimization procedure. The addition of terminal conditions makes the optimization problem similar in nature to the two-point boundary value problem, as noted above. Viewed in this context, endogenous time-horizon optimization is a form of two-point boundary value problem with the terminal point not fixed.

Specifying intervals of stabilization policy in this way, as well as using the associated terminal condition and endogenous time-horizon techniques, is not a universally applicable procedure. It is, however, suitable for the application of optimization analysis to short-term stabilization, defined, as above, as the restoration of the economy to its "normal, long-run acceptable" path after a deviation from that path.

In this context, it is important to distinguish the problem of stabilizing the economy, during deviations from a long-run path, from the problem of directing the economy from one long-run path to a different one. ${ }^{14}$ Methods designed for the former problem may not suffice for the latter. In particular, in the long-run case the time horizon chosen becomes the dominant element in the solution; and the policy interval itself emerges as an artificially contrived deviation from the original long-run path, ${ }^{\mathbf{1 5}}$ perhaps unmaintainable without an infinite time horizon to policy.

This distinction serves to emphasize the importance of the identification of the "normal, long-run acceptable" path or state of the economy and

fashion; it is important to note that this terminal condition is only one of many possible formulations.

14 The long-run problem is that of Ramsey (1928) and the subsequent literature of optimal growth.

15 An example of the confusion of stabilizing the economy with directing it to a new long-run path is in Pack (1964). Pack tested Phillips's proportional, integral, and derivative policy rules for their potential ability to stabilize the 1953-54, 1957-58, and 1960-61 recessions. Because he used a gross national product target extrapolated at $3 \mathrm{~T} / 2$ percent from the peak of the 1953 recession, the actual 1960-61 experience was far enough from the target to render stabilization policy as such inadequate in his tests. His 1960-61 experiments in fact aimed to move the economy to a long-run path which it had left nearly a decade earlier, not to stabilize a specific short-term deviation. For this reason, his 1960-61 experiments failed to produce the interesting results which his 1953-54 and 1957-58 experiments showed. 


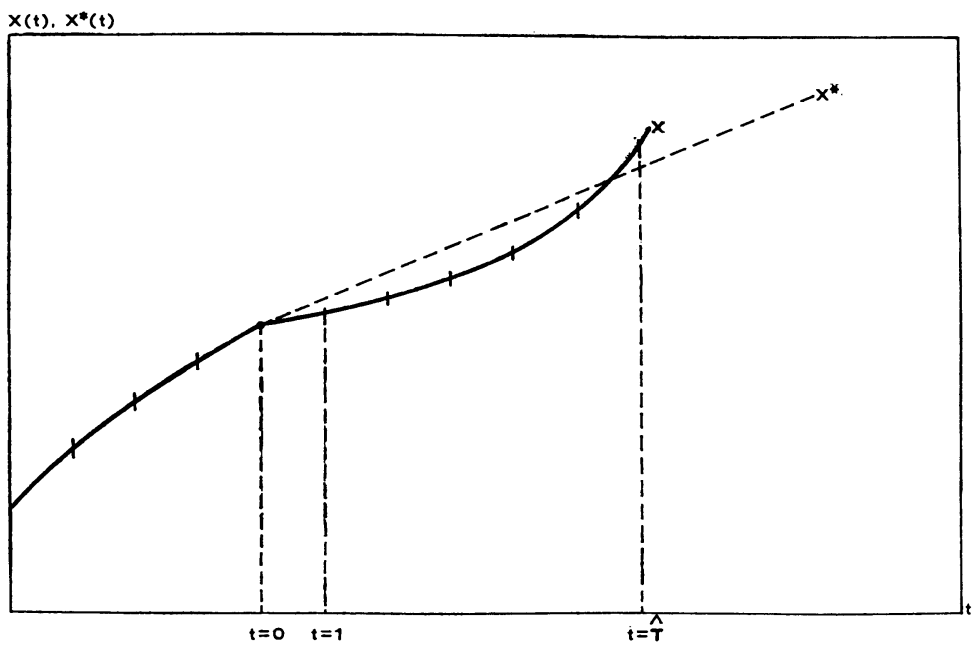

FIG. 4.-Recessionary policy interval based on variable $X$

the long-run policy which accompanies it. Such a path must be maintainable on an effectively permanent basis, that is, for the foreseeable future. Only in this context of short-run deviations about a self-maintaining ${ }^{16}$ long-run path does the policy-interval concept provide a workable approach to the formulation of stabilization policy. The techniques developed here, therefore, are suitable not for general application but only for a more restricted set of situations requiring the mitigation of short-term fluctuations. ${ }^{17}$

\section{The Solution Algorithm}

The algorithm that includes both the piecewise quadratic and the endogenous time-horizon extensions to the dynamic optimization model has a three-tiered structure which relies upon the technique, familiar in mathematical programming, of solving a complicated problem by breaking it down into a series of simpler problems. Level I is the classical Theil optimization with neither extension. Level II, which operates through

16 The long-run normal path, discussed in relation to the policy-interval concept, is self-maintaining in two senses. First, a long-run policy exists to move the economy along this path. Second, the inherent stability of the economy can contribute to correcting, in time, such deviations from the long-run path as do occur. The implied role for stabilization policy within a policy interval is, therefore, to correct these deviations more quickly and with less loss as measured by the given preference function.

17 Without holding categorically that the policy-interval approach is unsuitable for longer-run optimal growth exercises in the Ramsey tradition, one may argue that applying the approach developed here to such problems raises difficulties which the discussion here does not resolve. 
repeated solution of Level I problems in an iterative procedure, imposes upon the optimization the piecewise quadratic criterion function but uses an arbitrarily fixed time horizon. Level III, which operates through repeated solution of Level II problems in a similar iterative procedure, makes the time horizon endogenous and thereby completes the optimization.

The Level I problem, worked out in a readily computed form by Theil (1964, chap. 2), takes the following form:

Substituting (2) into (1) yields

$$
W(x)=k_{0}+k^{\prime} x+\frac{1}{2} x^{\prime} K x,
$$

where

$$
\begin{aligned}
& k_{0}=b^{\prime} s+\frac{1}{2} s^{\prime} B s, \\
& k=a+R^{\prime} b+\left(C+R^{\prime} B\right) s, \\
& K=A+R^{\prime} B R+C R+R^{\prime} C^{\prime} .
\end{aligned}
$$

Minimizing $W(x)$ with respect to $x$ yields the optimal solution

$$
\hat{\boldsymbol{x}}=-K^{-1} k \text {. }
$$

This solution satifies the first-order condition

$$
\frac{d W(x)}{d x}=0
$$

The necessity that $K$ be nonsingular, an important point below, follows from the second-order minimum condition that $K$ be positive definite. The optimal $\hat{y}$ follows from substituting $\hat{x}$ into (2). Hence, the Level I problem yields an optimal solution $(\hat{x}, \hat{y})$ and associated ${ }^{18}$ optimal $\tilde{x}$ and $\tilde{y}$.

It is important to note the dimensions of the vectors and matrices in this solution. The vector $x$ is a stacked vector; hence, for the case of $m$ instrument variables, the vector $x$ has $m T$ elements, where $T$ is the fixed time horizon for the Level I problem. Similarly, for the case of $n$ target variables, vectors $y$ and $s$ have $m T$ elements. All other vectors and matrices are conformable.

The Level II problem solves a series of Level I problems, each with different $\boldsymbol{A}$ and/or $\boldsymbol{B}$ matrices and a different $s$ vector.

On the first subiteration, ${ }^{19}$ the diagonal elements $a_{i i}$ assume, for those $x_{i}$

18 In Theil's standard quadratic case, the optimal $x$ and $y$ follow from the definitions $\tilde{\boldsymbol{x}}=\hat{\boldsymbol{x}}-\boldsymbol{x}^{*}$ and $\tilde{\boldsymbol{y}}=\hat{\boldsymbol{y}}-\boldsymbol{y}^{*}$. For a piecewise quadratic criterion function with nontrivial zero loss sets, the optimal $\tilde{\boldsymbol{x}}$ follow from decision rule (4), and similarly for the optimal $\tilde{y}$.

19 The repeated solutions of the Level I problem within a given Level II problem are called "subiterations"; "iterations" are the repeated solutions of the Level II problem within a given Level III problem. 
which have piecewise quadratic treatment, either the $a_{i i}{ }^{u}$ or the $a_{i i}{ }^{l}$ values; any number of arbitrary decision rules may suffice. The $x_{i}{ }^{*}$, to be used in calculating $\tilde{x}=x_{i}-x_{i}{ }^{*}$ on this subiteration, assume the values $x_{i}{ }^{u}$ to correspond to $a_{i i}{ }^{u}$ or $x_{i}$ to correspond to $a_{i i}{ }^{l}$. For those $x_{i}$ which do not bear piecewise quadratic treatment, the $a_{i i}$ and $x_{i}^{*}$ values are straightforward.

The $b_{i i}$ and $y_{i}{ }^{*}$ values for the first subiteration follow from the same arbitrary decision rule; and the $y_{i}{ }^{*}$ and $x_{i}{ }^{*}$ vectors, together with the other information necessary for the Level I problem, suffice to derive the $s$ vector ${ }^{20}$ for the first subiteration. Using these $\boldsymbol{A}$ and $\boldsymbol{B}$ matrices and this $s$ vector, the algorithm solves the Level I problem, yielding a set of optimal $\hat{x}_{i}$ and $\hat{y}_{i}$.

For any subiteration other than the first, the algorithm adjusts the $\left(a_{i i}\right.$, $\left.x_{i}{ }^{*}\right)$ and the $\left(b_{i i}, y_{i}{ }^{*}\right)$ according to decision rules (3) and (4), using the set of optimal $\left(\hat{x}_{i}, \hat{y}_{i}\right)$ from the previous subiteration. Having applied these decision rules, the algorithm uses the adjusted $\boldsymbol{A}$ and $\boldsymbol{B}$ matrices and the adjusted $s$ vector to resolve the Level I problem.

The Level II problem terminates on the first subiteration for which decision rules (3) and (4) call for no adjustment to be made in any $\left(a_{i i}\right.$, $\left.x_{i}{ }^{*}\right)$ or $\left(b_{i i}, y_{i}{ }^{*}\right)$. Any further subiterations would simply reproduce the final solution.

The Level III problem solves a series of Level II problems, each with a different trial time horizon.

On the first iteration, the trial time horizon $T$ is arbitrary. The algorithm then solves the Level II problem for fixed time horizon $T$. After solving the Level II problem, the algorithm checks the optimal $(\hat{x}, \hat{y})$ for satisfaction of the terminal conditions. There are three possibilities:

If $(\hat{x}, \hat{y})$ first satisfies the terminal conditions in $\hat{T}=T$, the Level III problem terminates. This $(\hat{x}, \hat{y}, \hat{T})$ is the final solution for the policy interval.

If $(\hat{x}, \hat{y})$ first satisfies the terminal conditions in $\hat{T}<T$, the algorithm resets $T=\hat{T}$ for the next iteration and again solves the Level II problem.

If $(\hat{x}, \hat{y})$ fails to satisfy the terminal conditions in any period up to and including $T$, the algorithm resets $T=T+1$ for the next iteration and again solves the Level II problem.

At this point, several observations are in order about the interaction among the three levels of the algorithm:

First, a qualification to the flexibility of the piecewise quadratic criterion function arises as a "nonredundancy" restriction on the number of the

20 The $s$ vector varies from one subiteration to the next, because it effectively normalizes constraints (2) about the particular $x_{i}^{l}$ or $x_{i}^{u}$ being used for $x_{i}^{*}$ and the particular $y_{i}^{l}$ or $y_{i}{ }^{u}$ being used for $y_{i}^{*}$. To be explicit, the algorithm involves rewriting constraints (2) as $\tilde{\boldsymbol{y}}=\boldsymbol{R} \tilde{\boldsymbol{x}}+\widetilde{\boldsymbol{s}}$ and using this " $\widetilde{\boldsymbol{s}}$ " for $\boldsymbol{s}$. Since $\tilde{\boldsymbol{x}}$ and $\tilde{\boldsymbol{y}}$ vary (for given $x$ and $y$ ) according to decision rules (4), this " $\tilde{s}$ " varies also. 
$x_{i}$ and $y_{i}$ which can simultaneously bear the full piecewise quadratic treatment. Any number may have asymmetrical terms with $a_{i i}{ }^{u} \neq a_{i i}{ }^{l}$ or $b_{i i}{ }^{u} \neq$ $b_{i i}{ }^{l}$, so this restriction applies only to those terms with nontrivial sets $M\left(x_{i}\right)$ or $M\left(y_{i}\right)$, that is, with $x_{i}{ }^{u} \neq x_{i}{ }^{l}$ or $y_{i}{ }^{u} \neq y_{i}{ }^{l}$. The source of this restriction is the requirement that matrix $K$, as defined in (9), be nonsingular for the solution of the Level I problem in (10). If a sufficient number of $x_{i}$ and $y_{i}$ bear piecewise quadratic treatment with nontrivial $M\left(x_{i}\right)$ and $M\left(y_{i}\right)$, and if a particular relationship among these variables maintains via equation (2), then, in the event that certain $x_{i}$ fall in $M\left(x_{i}\right)$ and certain $y_{i}$ fall in $M\left(y_{i}\right)$ simultaneously, matrix $K$ will have one or more rows and columns consisting of zero vectors. More specifically, each column of matrix $K$ corresponds to one $x_{i}$, the value of a particular instrument variable in a particular time period. If the optimal $\hat{x}_{i}$ falls in $M\left(x_{i}\right)$, then that $\hat{x}_{\mathrm{i}}$ depends only upon the effect of movements in $x_{i}$ on the target variables in the problem. If, in addition, however, all $y_{i}$ affected by $x_{i}$ via equation (2) fall in their respective $M\left(y_{i}\right)$, then the $K$ matrix will apply only zero elements to values of $x_{i}$. Hence, the value of $\hat{x}_{i}$ under such circumstances is indeterminate, as illustrated operationally by the singularity of $K$. The nonsingularity or "nonredundancy" restriction in the Level II problem is a prohibition barring any pattern of piecewise quadratic terms which could yield such a result in an associated Level I problem. ${ }^{21}$

A second restriction is that any variable which is a part of the terminal conditions in the Level III problem must be treated in the piecewise quadratic mode in the Level II problem. Suppose, for example, that in the example of Section II, the only terminal condition is the restoration of real output to the minimum desired level. In the absence of piecewise quadratic treatment, those elements $y_{i}^{*}$ which correspond to successive periods' values of real output assume, as fixed values, the successive minimum desired levels. Then a problem arises in that the Level I problem penalizes not only values of real income below the minimum desired level, but any value above this level as well; this effect retards satisfaction of the terminal condition, with distorting effects for the Level III problem. In general, piecewise quadratic treatment of the terminal-condition values is necessary to provide a zero-penalty region on the appropriate side of the relevant $\left(x_{i}^{*}, y_{i}^{*}\right)$. Hence, piecewise quadratic optimization, although it is an entirely independent generalization of the Tinbergen-Theil approach, is a necessary precursor to the application of endogenous time horizon optimization to economic stabilization policy.

A third major problem, left unanswered in the exposition of the Level

21 In practice, this restriction does not seem cumbersome. Any piecewise quadratic function which sets $x_{i}{ }^{*}=x_{i}{ }^{u}=x_{i}{ }^{l}$ for all $x_{i}$ satisfies the restriction regardless of the presence of nontrivial $\boldsymbol{M}\left(y_{i}\right)$. In cases for which a nontrivial $M\left(x_{i}\right)$ is essential for one or more $x_{i}$, reference to the $\boldsymbol{R}$ matrix indicates the implied restrictions for the associated $M\left(y_{i}\right)$. 
III problem above, is the possibility that the optimal $(\hat{x}, \hat{y})$ may satisfy the terminal conditions for no reasonable value of $T .{ }^{22} \mathrm{~A}$ terminal condition involving 2 percent unemployment and zero price inflation, for example, is unlikely to be satisfied in the context of any reasonable model of the economy. It is this problem which effectively restricts the applicability of the policy interval concept and the associated algorithm to short-term stabilization policy, as the discussion of Section II indicates.

\section{A Computational Example}

The 1957-58 recession in the United States may serve as a good example of a policy interval to illustrate the application of the methods developed above. The model of the economy used is the Wharton model (Evans and Klein 1968), linearized so as to reflect the behavior of the economy in the $1957-58$ period. ${ }^{23}$

The 1957-58 recession has been, to date, the most severe in the postwar period. Real output fell from a peak of $\$ 455.2$ billion $^{24}$ in 1957:III (third quarter of 1957) to $\$ 437.5$ billion in 1958 :I, for a decline of nearly 4 percent. Not until 1958:IV did real output regain its prerecession peak value. At the same time, the total unemployment rate rose from 4.0 percent in 1957:I to over 7.4 percent, the highest value observed in the postwar period, in 1958:III. To date 1958:II and 1958:III have been the only quarters in the postwar period to register a total unemployment rate above 7 percent.

In the terminology of Section II, the 1957-58 experience constitutes an easily recognizable recessionary policy interval. Nevertheless, it is difficult, especially in the context of the purely expository aim of this section, to arrive at clearly satisfactory initial and terminal conditions for this policy interval. The unemployment rate first began to rise in 1957:II, but the increase was not pronounced until 1957:IV, and the unemployment rate did not go above 5 percent until 1958:I. Real output reached a clear peak in 1957:III, but this peak represented a growth of less than 2 percent at an annual rate since the previous quarter, and the level of real output was lower in 1957:II than in 1957:I. Hence, in the absence of a clear definition of the policy of the period-as seems always the case in historical exercises -no straightforward initial condition for the policy interval is obvious. ${ }^{25}$

22 This point concerns the convergence properties of the Level III problem. For a discussion of these convergence properties, as well as those of the Level II problem, see Friedman (1971), chaps. 6 and 7.

23 The motivation and technique of the linearization are discussed in Friedman (1971), chaps. 2 and 3.

24 Values for output and government purchases, stated in this section, are seasonally adjusted quarterly values at annual rates. Price and unemployment values are also seasonally adjusted. Data are from Survey of Current Business; The National Income and Product Accounts of the United States, 1929-1965; and Federal Reserve Bulletin.

25 The discussion in Section II of the function of initial conditions in the policy- 
TABLE 1

Nonconstant $x^{*}$ and $y^{*}$ Values

\begin{tabular}{|c|c|c|c|c|c|}
\hline & \multirow[b]{2}{*}{ Quarter } & \multicolumn{2}{|c|}{$G$} & \multicolumn{2}{|c|}{$X$} \\
\hline & & $G_{t}^{l}$ & $G_{t}^{u}$ & $X_{t}^{l}$ & $X_{t}^{u}$ \\
\hline 1957:III & $\ldots \ldots \ldots$ & 84.1 & 89.1 & 456.6 & 466.6 \\
\hline 1957:IV & (n) & 84.9 & 89.9 & 459.9 & 470.1 \\
\hline 1958:I. & & 86.8 & 91.8 & 463.4 & 473.6 \\
\hline 1958:II & & 88.6 & 93.6 & 466.8 & 477.1 \\
\hline 1958:III & & 89.8 & 94.8 & 470.3 & 480.6 \\
\hline 1958:IV & & 91.5 & 96.5 & 473.7 & 484.2 \\
\hline
\end{tabular}

Note.- $-G=$ federal government purchases, $\$ 1958$ billion; $X=$ gross output, $\$ 1958$ billion.

A compromise solution to this problem is to select real output as the primary indicator of a recessionary policy interval. A subjective view of the historical period suggests 1957:III as an arbitrary initial quarter of the policy interval, and a terminal condition which requires that real output $(X)$ equal or exceed its minimum acceptable level, specified as a 3 percent per annum projection of the level of real output in 1957:II. Table 1 shows the quarterly values of this path.

The criterion function assigns a loss to any value of real output below the relevant minimum acceptable level. There is no reason to assign losses to all values above this level, however, and so it is necessary to treat real output in a piecewise quadratic function. Hence, table 1 labels the projection from the observed $\$ 453.2$ billion in 1957:II as $X_{t}{ }^{l}$, because these values divide the sets $L\left(X_{t}\right)$ and $M\left(X_{t}\right)$.

Choosing the corresponding $X_{t}{ }^{u}$, to divide the sets $M\left(X_{t}\right)$ and $U\left(X_{t}\right)$, is an entirely arbitrary matter. Table 1 shows $X_{t}^{u}$ values which are a projection, at the same 3 percent annual rate used for the $X_{t}{ }^{l}$, from a base level $\$ 10$ billion above the actual value of real output in 1957:II. Since the sample exercise presented here treats a recessionary policy interval, the $X_{t}{ }^{u}$ values would be relevant only in the case of extreme overshooting. This situation does not in fact arise, so the $X_{t}^{u}$ values are largely superfluous; the sets $M\left(X_{t}\right)$ could just as well be unbounded above.

To summarize, the policy interval in this exercise begins in 1957:III and terminates when the optimal value of real output $X_{t}$ in any period satisfies $X_{t} \in M\left(X_{t}\right)=\left\{X_{t} \mid X_{t}{ }^{l} \leqslant X_{t} \leqslant X_{t}{ }^{u}\right\}$. The exercise focuses on five variables: three target variables-real output, the gross national product price deflator, and the total unemployment rate; and two instrument variables - total government purchases and the central bank discount rate. ${ }^{26}$ Table

interval approach suggests both the difficulty and the nonnecessity of specifying such conditions precisely in historical exercises.

26 It is possible to argue that the central bank discount rate is not an exogenous tool of monetary policy but, rather, an endogenous variable which follows market rates. Even under this view, there remain two related rationales for using the discount 
TABLE 2

ACtUal $x$ AND $y$ Values

\begin{tabular}{|c|c|c|c|c|c|c|}
\hline & Quarter & $G$ & $I D$ & $X$ & $P$ & $U N$ \\
\hline 1957:III & 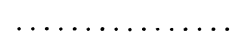 & 89.1 & 3.19 & 455.2 & 0.980 & 4.20 \\
\hline 1957:IV & & 89.9 & 3.25 & 448.2 & 0.985 & 4.97 \\
\hline 1958:I & $\ldots$ & 91.8 & 2.68 & 437.5 & 0.993 & 6.27 \\
\hline 1958:II & & 93.6 & 1.84 & 439.5 & 0.997 & 7.30 \\
\hline 1958:III & & 94.8 & 1.80 & 450.7 & 1.001 & 7.43 \\
\hline 1958:IV & & 96.5 & 2.29 & 461.6 & 1.006 & 6.43 \\
\hline
\end{tabular}

Note.- $G=$ federal government purchases, $\$ 1958$ billion; $I D=$ discount rate, percentage; $X=$ gross output, $\$ 1958$ billion; $P=\mathrm{GNP}$ price deflator index, $1958=1.00 ; U N=$ total unemployment rate, percentage.

2 actually observed values of these variables. The description of the policy interval itself outlines the treatment of real output in this exercise. The treatment of the other four variables is as follows.

The desired path for the price deflator for gross national product $(P)$ is a constant vector with each element equal to 0.971 , the actual level in 1957:II.

The desired path for unemployment $(U N)$ is a constant 4.0 percent level.

The government purchases of goods and services variable $(G)$ bears piecewise quadratic treatment. The actual values, given in table 2, form the $G_{t}{ }^{u}$ path. ${ }^{27}$ The $G_{t}{ }^{l}$ path is a set of values, each of which is lower than the corresponding actual, or $G_{t}{ }^{u}$, value by $\$ 5$ billion. Table 1 shows the $G_{t}{ }^{l}$ and $G_{t}{ }^{u}$ paths.

The central bank discount rate $(I D)$ also bears piecewise quadratic treatment, with $I D_{t}{ }^{*}=I D_{t}{ }^{l}=I D_{t}{ }^{u}$ constant at 3.0 percent. Hence, the zero loss ranges $M\left(I D_{t}\right)$ exist only trivially as points. The piecewise quadratic treatment arises in the differential criterion function weighting pattern for sets $L\left(I D_{t}\right)$ and $U\left(I D_{t}\right)$.

The criterion function in this exercise assigns loss values only to squared deviations of the target and instrument variables and not to cross-products of deviations; that is, the criterion function vectors $a$ and $b$ are null vectors, matrices $\boldsymbol{A}$ and $\boldsymbol{B}$ are diagonal matrices, and matrix $\boldsymbol{C}$ is a null matrix, as in the simplified exposition of Section I. The weighting patterns for all five variables are as follows. ${ }^{28}$

rate as an instrument variable. First, even though it follows market rates, it bears an announcement effect which influences expectations by confirming Federal Reserve approval of financial market trends and intentions of maintaining them. Second, it may at times serve as a more general proxy for shifting monetary policy stances which are otherwise difficult to quantify.

27 Any such use of actual values implies some assumption that expenditure policy at the time corresponded to social needs evaluated independently of any need for antirecessionary fiscal policy.

28 This paper presents no arguments to defend the particular criterion function 
TABLE 3

Optimal $\hat{\boldsymbol{x}}$ and $\hat{\boldsymbol{y}}$ Values

\begin{tabular}{|c|c|c|c|c|c|c|}
\hline & Quarter & $G$ & $I D$ & $X$ & $P$ & $\overline{U N}$ \\
\hline 1957:III & 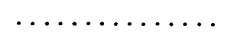 & 89.7 & 2.95 & 456.5 & 0.980 & 3.99 \\
\hline 1957:IV & $\ldots \ldots \ldots$ & 95.4 & 2.98 & 459.8 & 0.986 & 3.10 \\
\hline 1958:I . & $\ldots$ & 102.8 & 2.99 & 463.1 & 0.996 & 2.22 \\
\hline 1958:II & & 104.0 & 3.00 & 466.6 & 1.002 & 3.18 \\
\hline 1958:III & & 101.9 & 3.00 & 470.2 & 1.007 & 4.72 \\
\hline 1958:IV & & 102.1 & 3.00 & 475.0 & 1.012 & 4.81 \\
\hline
\end{tabular}

Note.--See note to table 2 for explanation of terms.

The piecewise quadratic weights for $X$ assign $b_{i i}{ }^{l}=50$ for $X_{t} \epsilon L\left(X_{t}\right)$ and $b_{i i}{ }^{u}=5$ for $X_{t} \epsilon U\left(X_{t}\right)$, reflecting the priority of the recessionary policy interval. The quadratic weight ${ }^{29}$ for $P$ is $b_{i i}=3.0 \times 10^{6}$. The quadratic weight for $U N$ is $b_{i i}=25$. The piecewise quadratic weights for $G$ assign $a_{i i}{ }^{l}=1.5$ for $G_{t} \epsilon L\left(G_{t}\right)$ and $a_{i i}{ }^{u}=1$ for $G_{t} \epsilon U\left(G_{t}\right)$. The piecewise quadratic weights for $I D$ assign $a_{i i}{ }^{l}=200$ for $I D_{t} \epsilon L\left(I D_{t}\right)$, reflecting the effective imposition of the constraint $I D \geqslant 3.0$, and $a_{i i}{ }^{u}=20$ for $I D_{t}$ $\epsilon U\left(I D_{t}\right)$.

The algorithm converges to $\hat{T}=6$ regardless of the initial arbitrary $T$. On the final iteration, three subiterations are required for the piecewise quadratic convergence. The criterion function, evaluated for the actually observed $x$ and $y$ over the interval, has value $5.34 \times 10^{4}$; evaluated for the optimal $\hat{x}$ and $\hat{y}$ over the interval, its value is $9.83 \times 10^{2}$. Table 3 shows the optimal $\hat{x}$ and $\hat{y}$.

It is clear from a comparison of tables 2 and 3 that the main effect of the optimization is to raise government purchases and, in so doing, to raise real output. The optimal $\hat{X}_{t}$ are consistently above the actual $X_{t}$; the difference exceeds $\$ 25$ billion in 1958: I, the trough quarter of the actual recession. In 1958: IV,$\hat{X}_{t}>X_{t}^{l}$ to yield time horizon $\hat{T}=6$ for the policy interval.

The increase in $X$ in the optimal solution is possible only at the cost of additional price inflation, however, and the optimal $\hat{P}_{t}$ are somewhat above the actual $P_{t}$, with the difference growing to 0.006 by the end of the policy interval. The narrowness of this margin indicates the unresponsiveness of prices in the Wharton model. The performance of the optimal $\widehat{U N_{t}}$ is superior to that of the actual $U N_{t}$.

The force moving the economy from the historical base to the optimal path is fiscal policy, operating through massive government purchases. The

weights chosen, and none is intended. The object of this exercise is simply to illustrate the operation of the piecewise quadratic and endogenous time-horizon optimization methods.

29 Because of the different units of measurement of the various $x$ and $y$, the $a$ and $\boldsymbol{b}$ values may be deceptive; $b_{i i}=3.0 \times 10^{6}$ for the price index does not represent a very large weight. 
optimal $\hat{G}_{t}$ are greater than the actual $G_{t}$ by more than $\$ 10$ billion in the two quarters of and immediately following the trough of the actual recession. The large $a_{i i}{ }^{l}$ placed on the discount rate effectively prevents that variable from falling significantly below its $I D_{t}{ }^{l}$ boundary, thereby providing a good example of the use of the piecewise quadratic criterion function to impose an inequality constraint on the optimization.

\section{Summary}

The piecewise quadratic criterion function generalizes the standard quadratic so as to facilitate a much more reasonable representation of economic policy preferences; its asymmetrical property also provides a ready method for imposing inequality constraints upon the optimization. Endogenous time-horizon optimization averts the arbitrariness of the selection of the time horizon in previous optimization methods, thereby permitting the optimization to deal with questions involving the desired speed of economic recovery; it also facilitates dealing with terminal conditions in the sense of dynamic programming. These two methods are independent in motivation but not in operation, in that the solution algorithm presented makes the piecewise quadratic criterion function a necessary precursor to endogenous time-horizon optimization. The combined effect of these two extensions to the Tinbergen-Theil model is to provide a framework which is not only sufficiently broad and flexible to treat many of the basic problems of formulating quantitative economic stabilization policy, but is also sufficiently straightforward in its operational and computational aspects to render such a treatment easily accessible for applied policy work.

\section{References}

Bellman, Richard E., and Dreyfus, Stuart E. Applied Dynamic Programming. Princeton, N.J.: Princeton Univ. Press, 1962.

Bryson, Arthur E., and Ho, Yu-Chi. Applied Optimal Control: Optimization, Estimation and Control. Waltham, Mass.: Blaisdell, 1969.

Evans, Michael K., and Klein, Lawrence R. The Wharton Econometric Forecasting Model. 2d ed. Philadelphia: Univ. Pennsylvania, Wharton School of Finance and Commerce, 1968.

Fan, Liang-Tseng, and Wang, Chiu-Sen. The Discrete Maximum Principle. New York: Wiley, 1964.

Fox, Karl A.; Sengupta, Jati K.; and Thorbecke, Lorik. The Theory of Quantitative Economic Policy with Applications to Economic Growth and Stabilization. Amsterdam: North-Holland, 1966.

Friedman, Benjamin M. Methods in Optimization for Economic Stabilization Policy. Ph.D. dissertation, Harvard Univ., 1971.

Hickman, Bert G., ed. Quantitative Planning of Economic Policy. Washington: Brookings Inst., 1965.

Mangasarian, Olvi L. Nonlinear Programming. New York: McGraw-Hill, 1969.

Pack, Howard. Formula Flexibility: A Quantitative Appraisal. Ph.D. dissertation, Mass. Inst. Tech., 1964. 
Pontryagin, L. S.; Boltyanskii, V. G.; Gamkrelidze, R. V.; and Mishchenko, E. F. The Mathematical Theory of Optimal Processes. New York: Wiley, 1962.

Ramsey, Frank P. "A Mathematical Theory of Saving." Econ. J. 38 (December 1928): 543-59.

Sage, Andrew P. Optimum Systems Control. Englewood Cliffs, N.J.: PrenticeHall, 1968.

Simon, Herbert A. "Dynamic Programming under Uncertainty with a Quadratic Criterion Function." Econometrica 24 (January 1956) : 74-81.

Theil, Henri. "A Note on Certainty Equivalence in Dynamic Planning." Econometrica 25 (April 1957): 346-49.

. Optimal Decision Rules for Government and Industry. Amsterdam: North-Holland, 1964.

Tinbergen, J. Economic Policy: Principles and Design. Amsterdam: North-Holland, 1966.

Zangwill, Willard I. Nonlinear Programming: A Unified Approach. Englewood Cliffs, N.J.: Prentice-Hall, 1969. 